\title{
ABSTRACTS
}

Meetings of The Keio Medical Society

\section{Engineering Fat Cell Fate to Fight Obesity and Metabolic Diseases}

\author{
Shingo Kajimura \\ UCSF Diabetes Center and Department of Cell Tissue Biology, San Francisco, USA
}

All mammals harbor two types of adipose tissues that serve distinct physiological functions: white adipose tissue (WAT) and brown adipose tissue (BAT). WAT functions mainly in the storage of excess energy, while BAT specializes in dissipating energy in the form of heat and functions as a defense against hypothermia and obesity. Since adult humans possess significant amounts of active BAT depots and it's mass inversely correlates with adiposity, BAT plays an important role in human obesity and energy homeostasis.

New evidence suggests two types of thermogenic adipocytes with distinct developmental and anatomical features: classical brown adipocytes and beige adipocytes. Classical brown adipocytes are located mainly in dedi- cated BAT depots of rodents and infants. Beige adipocytes, on the other hand, reside mainly in subcutaneous WAT where they arise postnatally in response to certain external cues, such as chronic cold exposure and longterm treatment with PPAR- agonists, a process often referred to as the "browning" of WAT. Importantly, adult human BAT appears to be mainly composed of beige-like adipocytes, making this cell type an attractive therapeutic target for obesity and obesity-related diseases, such as insulin resistance and type 2 diabetes. I will review recent progress in the molecular control of brown and beige adipocyte development and discuss emerging questions. (Presented at the 1912nd Meeting, December 21, 2015) 